\title{
Philosophiques
}

\section{Épistémologie génétique, science et philosophie}

\section{Maurice Gagnon}

Volume 4, numéro 2, octobre 1977

Philosophie et psychologie

URI : https://id.erudit.org/iderudit/203073ar

DOI : https://doi.org/10.7202/203073ar

Aller au sommaire du numéro

Éditeur(s)

Société de philosophie du Québec

ISSN

0316-2923 (imprimé)

1492-1391 (numérique)

Découvrir la revue

Citer cet article

Gagnon, M. (1977). Épistémologie génétique, science et philosophie.

Philosophiques, 4(2), 225-244. https://doi.org/10.7202/203073ar d'utilisation que vous pouvez consulter en ligne.

https://apropos.erudit.org/fr/usagers/politique-dutilisation/ 


\title{
ÉPISTÉMOLOGIE GÉNÉTIQUE, SCIENCE ET PHILOSOPHIE
}

\author{
par Maurice Gagnon
}

Le lecteur de Piaget ne peut s'empêcher de se poser des questions sur les rapports entre psychologie génétique et épistémologie génétique. Comment faut-il qualifier les incursions de Piaget dans des disciplines aussi diverses que la physique, la logique, la biologie et les mathématiques? Les rapprochements qu'il tente entre certains contenus de ces dernières et les données de la psychologie génétique sont-ils valables? Et que dire des notions que Piaget extrapole à partir de la psychologie génétique sur la connaissance en général? Et les questions qui précèdent nous amènent à nous en poser une autre : Piaget a-t-il réussi à construire une épistémologie scientifique clairement affranchie de la philosophie?

Nous allons, dans les pages qui suivent, exposer ce qui nous semble être les traits essentiels de la pensée piagétienne et de ses fondements, et tenter d'esquisser une réponse à ces questions.

\section{LES AVATARS DE L'ÉPISTÉMOLOGIE PHILOSOPHIQUE}

Les recherches de Piaget en épistémologie génétique furent à l'origine inspirées par le sentiment que les discussions épistémologiques entre philosophes, par exemple la bataille entre idéalistes et réalistes, ne pouvaient aboutir qu'à un cul-de-sac parce que les philosophes manquent d'information empirique concernant les processus d'acquisition de la connaissance, et parce qu'ils font des analyses trop superficielles de l'information très pauvre dont ils disposent. De plus, un système philosophique est, pour Piaget, une coordination de toutes les valeurs impliquées dans la vie humaine, et l'épistémologie y est un élément parmi d'autres. La construction d'une épistémologie philosophique est souvent plus fortement déterminée par le désir conscient ou inconscient du philosophe de l'ajuster aux autres parties du système, que par un examen attentif des différents types de connaissance qui existent de fait à une 
époque donnée. Une autre raison invoquée par Piaget pour rejeter l'épistémologie philosophique est que la philosophie n'est pas une science : les énoncés philosophiques sont trop généraux et trop vagues pour être effectivement falsifiables, car leurs conséquences ne peuvent être clairement infirmées par des contre-exemples précis.

\section{II. ÉPISTÉMOLOGIE SCIENTIFIQUE ET PSYCHOLOGIE GÉNÉTIQUE}

Piaget a fait le pari qu'une épistémologie scientifique est possible. Cette épistémologie doit d'abord être descriptive, c'est-àdire être fondée sur la pratique réelle des divers modes de connaissance, sur la façon dont la connaissance est effectivement acquise. Pour réaliser cet objectif, l'épistémologue devra diviser son sujet et en délimiter les parties, cesser d'aspirer comme le philosophe à la connaissance totale, éviter les systématisations hâtives pour raffiner plutôt l'analyse grâce à l'usage de méthodes rigoureuses dont les résultats précis et vérifiés entraînent l'accord des esprits. Deuxièmement, l'épistémologie scientifique sera comparative : comme il existe plusieurs types de connaissance et plusieurs méthodes pour acquérir la connaissance, l'épistémologie doit les analyser toutes en soulignant leurs différences et en les situant les unes par rapport aux autres selon des points de vue variés : par exemple, leur degré d'usage des mathématiques, ou certaines propriétés de leur conclusion, cumme l'exactitude, la probabilité, la précision et la falsifiabilité, etc...

En troisième lieu, l'épistémologie scientifique doit être génétique. Elle ne peut s'occuper exclusivement des contenus et des méthodes de la connaissance telle qu'elle existe à un moment donné. Analyser ces contenus et ces méthodes est assurément une tâche importante qui doit être accomplie. Mais ceci est insuffisant parce que la connaissance, surtout la connaissance scientifique, n'est pas une entité statique et immobile : elle apparaît plutôt comme un processus illimité de changement. Tout contenu cognitif peut être considéré comme une structure, c'est-à-dire comme un réseau de relations reliant entre eux un ensemble d'éléments. Chaque structure est l'aboutissement d'une genèse qui a eu comme point de départ une structure antérieure. En fait, la structure est une coupe plus ou moins artificielle et arbitraire dans le devenir continu de la 
connaissance. Il est donc normal pour un épistémologue d'analyser les différences qui existent entre la structure et les processus actuels d'une connaissance et certaines de ses structures et processus passés, et de se demander pourquoi et comment les dernières ont été abandonnées en faveur des premières. Piaget défend ici une idée qui a joué un rôle très important dans l'oeuvre de Gaston Bachelard, de Thomas Kuhn et d'Alexandre Koyré ${ }^{1}$.

Piaget considère l'histoire des sciences, qu'il a d'ailleurs enseignée pendant plusieurs années, comme une base indispensable de l'épistémologie génétique : elle fournit les matériaux sur lesquels cette dernière travaille. Mais l'épistémologie génétique de Piaget s'enrichit d'un autre fondement très particulier et original, soit la psychologie génétique. L'épistémologue, dit Piaget, doit examiner la connaissance dite vulgaire ou du sens commun, pour au moins deux bonnes raisons. D'abord, elle constitue le premier lieu universel de la connaissance, et plusieurs notions d'abord acquises et élaborées à ce niveau sont ensuite critiquées ou raffinées par la science. En second lieu, les épistémologies philosophiques se sont souvent préoccupées exclusivement ou surtout de la connaissance du sens commun. Il s'ensuit qu'une analyse exhaustive et précise de cette connaissance peut être utile pour clarifier les débats sans fin des épistémologies philosophiques.

La connaissance du sens commun commence à se construire dès les premiers mois de l'enfance; d'où la nécessité d'analyser le processus de formation de la connaissance au cours de cette période. Pour ce faire, Piaget a développé une méthode par laquelle l'enfant est placé dans une situation donnée, et est invité à résoudre un problème ou à répondre à une question à l'intérieur de la situation ; après, on lui demande une justification de sa solution ou de sa réponse. Ainsi, l'enfant révèle indirectement sa propre compréhension de la situation, et les concepts, s'il en existe, qui sont impliqués dans cette compréhension. Évidemment. les expériences sont nombreuses et variées quant à leur contenu, et chacune est répétée avec plusieurs enfants d'âges divers de sorte que l'évolution intellectuelle de l'enfant sera enregistrée. Dans le cas d'enfants trop jeunes pour utiliser le langage, leur comportement dans la situation donnée doit être prudemment interprété par le chercheur.

Jean PIAGET, Psychologie et épistémologie, Paris, Éditions Gonthier, 1970, pp. 110-130. 
Par cette méthode, Piaget espère trouver une meilleure réponse à certaines questions épistémologiques, comme par exemple : la connaissance dépend-elle de certaines entités innées à l'esprit, ou bien est-elle simplement une généralisation exprimant les éléments communs des objets découverts dans une analyse attentive de l'expérience?

Bien sûr, cette méthode ne peut produire de résultats valables si son usage n'est pas accompagné d'une bonne dose de prudence. Il est important de distinguer entre investigation psychologique et pédagogie, et de questionner les enfants d'une façon qui ne leur suggère en rien une réponse. Dans l'interprétation des réponses, le point de vue de l'observateur ne doit pas être confondu avec celui du sujet : c'est-à-dire que l'investigateur doit éviter de postuler la possession et l'usage par le sujet de catégories et de concepts propres à l'adulte. Ici, Piaget adopte le rasoir d'Occam comme règle méthodologique: les seules entités qu'on peut postuler comme éléments de la connaissance enfantine sont celles sans lesquelles le comportement observé du sujet, de même que les explications et justifications qu'il en donne, seraient impossibles ; et ces éléments postulés ne sont pas nécessairement semblables à ceux de la connaissance de l'adulte : il y a de très bonnes raisons de penser qu'ils sont différents.

Piaget vise à dévoiler ainsi l'ontogenèse de la connaissance, par opposition à la phylogenèse que constitue l'histoire des sciences. Cette ontogenèse se distingue également de l'épistémologie des sciences, qui est une anatomie comparée des structures, des processus et des cheminements de la science. La première joue par rapport à la seconde un rôle analogue à celui de l'embryologie visà-vis de l'anatomie comparée et de la théorie de l'évolution en biologie. On sait que l'embryologie a fourni de nouveaux arguments à la théorie de l'évolution en montrant que des espèces biologiques différentes se ressemblent davantage au stade embryonnaire qu'aux stades ultérieurs, mettant ainsi en lumière des homologies organiques auparavant inconnues ${ }^{2}$. Selon les termes mêmes de Piaget, la psychologie génétique peut " nous éclairer sur la véritable portée et sur les liaisons effectives des intuitions fondamentales, dont l'évolution des notions scientifiques a été, soit la

2. Jean PIAGET, Op. cit., pp. 33-38, 129-130. 
bénéficiaire, soit la victime $n^{3}$. Il est à noter que dans ce dernier cas, la psychologie génétique pourrait nous renseigner davantage sur les " obstacles épistémologiques" dont parlait Bachelard, obstacles issus de la connaissance vulgaire et nuisant au développement de la pensée scientifique.

\section{CARACTÈRES DE L'ÉVOLUTION INTELLECTUELLE DE L'ENFANT}

Piaget divise le développement intellectuel de l'enfant en trois périodes, soit la période sensori-motrice ( 0 à 2 ans), la période des opérations concrètes ( 2 à 11 ans) et la période des opérations formelles (11 à 14 ans). Chaque période est subdivisée en un certain nombre de stades. Voici les principaux traits de ces stades :

1) En ce qui concerne l'évolution des notions physiques, logiques et mathématiques, l'ordre de succession des stades est uniforme, indépendamment du milieu culturel, psycho-social et physique ; et chaque sujet les parcourt intégralement, sans en sauter un seul. Cette uniformité est garantie par les résultats communs de diverses études faites dans diverses régions du monde : Suisse, Iran, Hong-Kong, Martinique, Afrique du Sud, etc. Partout ces études ont confirmé la constance de la succession des stades, bien que le rythme de cette succession soit plus lent dans certains cas : le milieu social, familial, ou scolaire accélère ou retarde le développement ${ }^{4}$. Bien entendu, ceci ne s'applique pas aux notions morales, religieuses et sociologiques, dont le développement est davantage influencé par le milieu ambiant ${ }^{5}$.

2) Ils sont intégratifs : cela signifie que toujours certains éléments de la connaissance propres à un stade sont intégrés dans le stade suivant. Le devenir de la connaissance n'implique pas la destruction entière du contenu cognitif précédent, mais la préservation de certaines de ses parties aussi bien que leur transformation en éléments d'un contenu cognitif nouveau, plus vaste, plus complexe

3. Jean PIAGET, Op. cit., pp. $129 \cdot 130$.

4. Jean PIAGET; Op. cit, pp. 68-79.

5. Jean PIAGET; Le jugement moral chez l'enfant, 3e éd., Paris, P.U.F., 1969, p. 208 : " La croyance en la justice immanente provient d'un transfert sur les choses des sentiments acquis sous l'influence de la contrainte adulte." 
et plus extensif. Ici, Piaget développe la notion d'abstraction réfléchissante, dont les caractères principaux sont les suivants :

a) elle est pratiquée non pas à partir d'objets tels que contemplés par un pur spectateur (ce qui serait la notion classique d'abstraction), mais plutôt à partir des actions du sujet sur les objets et des réactions et transformations de ces derniers consécutives à ces actions. Si on centre son attention sur les transformations des objets, on obtient une connaissance empirique; si on considère plutôt les actions et leurs coordinations entre elles, on obtient une pensée logique et mathématique. Évidemment, l'abstraction réflexive est aussi une abstraction généralisatrice : elle isole les caractères communs d'entités singulières.

b) elle consiste en une sorte de retour critique, bien qu'inconscient, sur un contenu cognitif ou une représentation qui est devenu périmé en face de nouveaux faits ou entités rencontrés dans l'expérience. Certains éléments de cette représentation seront écartés, d'autres seront préservés mais aussi transformés à l'intérieur d'une nouvelle représentation qui peut contenir de nouvelles relations entre ces éléments, de même que des éléments entièrement neufs.

3) Une régression à des stades précédents peut arriver, mais elle est toujours temporaire et due à des difficultés d'adaptation à une situation d'expérience nouvelle et plus large.

L'uniformité de l'ordre de succession des stades implique l'existence d'un déterminisme dans le devenir intellectuel de l'enfant. Ce déterminisme résulte de la combinaison de plusieurs facteurs :

a) Le milieu familial, scolaire et social, dont l'influence positive ou négative va stimuler ou inhiber le développement de l'enfant.

b) La structure biologique de l'organisme, en particulier la structure sensori-motrice et neurologique, dont le cerveau est l'élément principal. Cette structure est le résultat de l'évolution biologique et elle constitue selon Piaget la seule condition innée de la connaissance parce qu'elle est transmise par les parents dans le processus de génération. Elle détermine partiellement la structure et les caractères de nos actions physiques sur les choses, actions qui constituent le point de départ de l'abstraction réfléchissante, donc de notre connaissance de ces 
choses. Une anomalie ou une insuffisance de l'équipement sensori-moteur est accompagnée par un retard ou une incapacité correspondante au niveau cognitif.

c) Le monde extérieur, qui détermine aussi partiellement la structure et les caractères de nos actions physiques, car ces dernières doivent, pour être couronnées de succès, s'adapter à l'objet sur lequel elles travaillent, ou qu'elles visent.

d) Piaget pense que les trois facteurs déjà mentionnés sont nécessaires, mais insuffisants. Il en ajoute un quatrième : l'activité d'équilibration accomplie par le sujet. Ce dernier tend à compenser les perturbations issues du monde extérieur en réalisant une coordination d'opérations, c'est-à-dire d'actions réversibles, coordonnées avec d'autres actions de manière à former un ensemble structuré ou système, et intériorisables. Une opération peut donc être physique ou purement mentale ou formelle. Les actions intelligentes sont définies comme des activités créant un état d'équilibre entre les actions du sujet et les réactions du monde extérieur à ces actions. L'intelligence serait ainsi un cas particulier de la fonction générale d'adaptation, définie comme la création d'un équilibre entre l'accommodation (transformation du sujet par le monde externe) et l'assimilation (transformation du monde externe par le sujet). Quand l'enfant devient adolescent et atteint le niveau des opérations formelles, l'équilibre couvre des situations des plus globales et complexes?.

Le déterminisme du développement intellectuel de l'enfant n'est pas surprenant si nous considérons que l'équipement biologique, neurologique et sensori-moteur standard est essentiellement le même pour la très grande majorité des enfants. Les cas anormaux sont relativement rares et facilement repérables à cause de la lenteur ou du plafonnement hâtif de leur capacité cognitive. De plus, les caractères spatiaux et qualitatifs de l'action de l'enfant sur les objets physiques extérieurs, de même que les réactions des

6. Une action est réversible quand il existe une autre action qui est son inverse. Par exemple, parcourir un espace, ou sérier des objets en allant du plus petit au plus gros sont des actions ré versibles; mais boire un verre d'eau ou fumer sa pipe n'en sont pas. Une action est intériorisable quand elle peut être accomplie en pensée seulement.

7. Jean PIAGET, Psycbologie et épistémologie, pp. 59-78; Six études de psycbologie, Paris, Éditions Gonthier, 1964, pp. 114-132, 172-173. 
objets à ces actions, sont partout les mêmes malgré les différences culturelles, géographiques, climatiques, etc . . En conséquence, le développement des premières notions biologiques, physiques, logiques et mathématiques est universellement le même chez les enfants. Il en va différemment, comme nous l'avons souligné plus haut, des notions religieuses, morales et sociologiques, dont le contenu est fortement déterminé par la culture du milieu ambiant.

\section{QUELQUES EXEMPLES DES RÉSULTATS DE LA PSYCHOLOGIE GÉNÉTIQUE ET LEURS RAPPORTS AVEC L'É- PISTÉMOLOGIE DES SCIENCES}

Je ne puis faire ici une description complète des résultats obtenus par Piaget. Mais qu'on me permette tout de même de les illustrer par quelques exemples.

\section{A. La causalité efficiente}

La causalité efficiente semble n'être rien de plus, pour l'enfant âgé de cinq mois, qu'un vague sentiment de l'efficacité de certaines de ses actions: l'enfant répète les mêmes gestes pour produire certains résultats obtenus d'abord par hasard. Mais il est sûrement inconscient des connections mécaniques impliquées dans la chaîne causale, étant donné qu'il essaie d'obtenir les mêmes résultats par les mêmes moyens, même quand certaines des connections ont été supprimées. Par exemple, il va agiter sa main fermée comme si la ticelle qui pendait du hochet placé au-dessus de sa couchette était encore là, et montrer sa surprise et sa déception parce que le hochet demeure immobile. Il essaie même d'obtenir le résultat désiré en agitant ses mains et ses pieds dans toutes les directions et en se cambrant ; et il montre de nouveau son désappointement en constatant l'inutilité de ses efforts.

Entre 5 et 24 mois, son expérience du monde environnant devient de plus en plus complexe et variée, et il devient peu à peu conscient de la nécessité des connections mécaniques et spatiales dans les chaînes causales, tout au moins dans un espace relativement proche de lui ; et il délègue le pouvoir causal à d'autres agents (v.g., il dirige la main de son père vers un objet qu'il veut lui faire prendre ou toucher). Plus tard, la spatialisation de la causalité est étendue aux objets éloignés dans l'espace ; et quand l'enfant a atteint l'adolescence et devient capable d'opérations for- 
melles, il tend à assimiler la causalité à la dépendance fonctionnelle et à la déduction logique. L'évolution de la causalité prend une direction humienne, et va même aller encore plus loin que chez Hume pour l'individu qui reçoit une éducation scientifique ${ }^{8}$.

Se fondant sur cette description de l'évolution de la notion de cause, Piaget contredit Hume sur deux points. D'abord, il soutient qu'il y a un fondement empirique réel de la notion de cause dans l'expérience tactilokinesthésique qui implique les actions de manipuler, pousser, lancer, tirer, modeler, etc . . . Ces actions sont accompagnées d'un sentiment d'efficacité, le sentiment d'imposer des transformations à des objets extérieurs. En second lieu, Piaget affirme que l'usage abusif du concept de cause ne dépend pas d'une naïve association d'idées, mais plutôt d'une extrapolation illégitime de certains caractères de notre expérience immédiate dans des objets qui sont très différents de nous, ou dans des séquences d'actions qui sont très différentes des nôtres. Surtout quand il n'y a pas de place pour des sensations d'effort, de poussée ou de résistance, ni même pour un contact entre des objets dans une séquence d'actions, l'usage du concept de cause est hautement discutable et il est épistémologiquement plus prudent de le remplacer par celui de dépendance fonctionnelle. Il semble inutile d'ajouter que Piaget rejette toute théorie absolument a priori de la causalité : si une notion ou une forme de la causalité était présente dans notre esprit dès le début, il n'y aurait pas de raison pour que la notion soit graduellement construite et reconstruite depuis la première enfance jusqu'à l'adolescence et même après, dans les cas où une éducation scientifique est acquise.

\section{B. La notion de classe}

La notion logique de classe se développe lentement à mesure que l'expérience du monde extérieur devient de plus en plus variée et précise. Avant l'âge de 7 ans, on n'a pas affaire à des classes, mais seulement à des collections, c'est-à-dire à des ensembles d'objets qui peuvent être tous perçus simultanément. Si une collection d'objets hétérogènes est étalée devant l'enfant et si on lui demande de grouper ensemble ceux qui sont "les mêmes ", l'enfant âgé de

8. Jean PIAGET, La construction du réel chez l'enfant, 4e éd., Neuchâtel, Delachaux et Niestlé, 1967, chap. 3. Lire aussi : Jean Piaget et Bärbel INHelder, De la logique de l'enfant à la logique de l'adolescent, Paris, P.U.F., 1970. 
$2 \frac{1}{2}$ à 5 ans arrange la collection totale en une figure géométrique quelconque (cette figure étant parfois une ligne droite) ou lui donne la forme d'un objet qui lui est familier. Ici, la collection est "figurale ". L'enfant essaie de regrouper dans la figure les objets qui sont semblables par la forme, la couleur, les dimensions, etc . . . Mais le critère de ressemblance n'est pas établi initialement pour la totalité entière ; l'enfant choisit un objet au hasard, puis un second et ainsi de suite. Il passe d'un critère de ressemblance à un autre ou de la ressemblance à la convenance, c'est-à-dire place un chien près d'un homme parce que le chien est l'ami de l'homme et le protège, ou bien un carrosse à côté d'un bébé parce que les carrosses sont faits pour les bébés, etc.

Plus tard, de 5 à 7 ans, l'enfant va commencer à subdiviser réellement la collection en sous-collections sans essayer de lui donner une forme générale. Le comportement précédemment observé concernant la ressemblance et la convenance persiste. $\mathrm{La}$ collection n'est plus figurale, mais c'est encore une collection.

Vers 7 ans, l'enfant commence à construire le concept de classe. Une classe est un groupe d'objets qui ont un quelconque prédicat commun, mais qui ne sont pas nécessairement tous perceptibles au même moment. L'enfant est maintenant capable de subdiviser correctement la classe en sous-classes en évitant ses erreurs précédentes sur la ressemblance et la convenance. Mais la compréhension correcte des rapports entre extension et compréhension, entre la classe et ses sous-classes, et entre "tous" et "quelques" n'est pas facile à atteindre. L'enfant a une forte tendance à confondre la classe entière avec l'une ou l'autre de ses plus grandes sous-classes : toutes les perles en bois feront un collier plus long que toutes les perles. Ceci se corrigera vers huit ou neuf ans. Concurremment, le concept de la classe nulle ou vide n'est atteint qu'à dix ans, celui de la classe unitaire à huit ans?

\section{Le nombre}

Le concept de nombre, avec ses deux aspects ordinal et cardinal, n'est pas construit avant l'âge de six ans, et Piaget interprète cette acquisition comme résultant de la fusion de deux ac-

9. Jean PIAGET et Bärbel INHELDER, La genèse des structures logiques élémentaires, Neuchâtel, Delachaux et Niestlé, lère éd., 1959, surtout les chapitres 1-5. 
quisitions antérieures, soit l'inclusion des collections les unes dans les autres, ou leur hiérarchisation les unes par rapport aux autres, par le moyen de la classification, et la sériation d'objets par la composition de relations asymétriques. Ces opérations sont d'abord concrètes, et non formelles : elles ne font pas abstraction d'un contenu qualitatif déterminé. Les classifications sont fondées sur les ressemblances et les différences entre les choses, mais principalement sur les ressemblances, et les objets sont ici considérés comme équivalents ; la sériation considère les objets plutôt comme différents, elle ordonne les différences selon une progression quelconque. Quand les notions de classe et de série deviennent formelles par le processus d'intériorisation, il devient possible de considérer les objets quelconques auxquels elles s'appliquent comme étant à la fois équivalents et différents : chaque objet est équivalent à n'importe quel autre, mais s'en distingue aussi par un ordre quelconque d'énumération ${ }^{10}$. Nous obtenons ainsi la série des nombres naturels.

Il est intéressant de confronter ces résultats avec les doctrines intuitionniste et logiciste concernant les fondements des mathématiques. D'une part, la construction de la notion de nombre présuppose, selon Piaget, celle des notions logiques de classe et de nonidentité, ce qui s'accorde avec la thèse logiciste selon laquelle les concepts mathématiques sont dépendants des concepts logiques. Mais cette dépendance n'est pas de nature axiomatique, contrairement à ce que prétendaient les logicistes. Les notions de classe et de non-identité sont simplement des éléments ou matériaux indispensables à la construction de la notion de nombre, qui est une synthèse des notions de classe et de série ordonnée. Ce résultat suppose l'expérience de nos actions sur les choses, avec leurs résultats et leurs coordinations entre elles, et ceci antérieurement à leur intériorisation. Il y a donc un élément intuitif à la base même de la connaissance arithmétique. Cet élément intuitif est contenu dans l'expérience de la classification et de la sériation effectuées par comparaison qualitative ; il a un contenu déterminé et n'est pas purement relationnel, c'est-à-dire logique ou formel. Il devient formel lorsqu'il est intériorisé, transpose sur un plan abstrait et indépendant des contextes particuliers. Mais il ne se ramène pas à

10. Jean PIAGET et Alina SzEMINSKA, La genèse du nombre chez l'enfant, Neuchâtel, Delachaux et Niestlé, 4e éd., 1967 ; Psychologie et épistémologie, pp. 18-21, 50-58. 
l'intuition intérieure du temps pur, ou à l'intuition de l'espace quantitatif et neutre, telles que nous les décrivent respectivement Brouwer et Bergson".

Une perspective génétique diffère cependant d'une perspective axiomatique, qui vise essentiellement la précision des concepts, leur ordonnance rigoureuse et la clarification de leurs relations mutuelles. Il peut arriver que les analyses génétiques et axiomatiques aboutissent aux mêmes résultats, mais comme elles poursuivent des objectifs différents, on ne saurait imposer aux secondes de s'aligner sur les premières. Même si Piaget avait énoncé au siècle dernier sa théorie de la genèse du nombre, et même si cette théorie avait été d'une évidence et d'une vérité indiscutables, elle n'aurait pas constitué pour Frege et Russel une raison valable de renoncer à leur entreprise logiciste. La même remarque vaut pour les axiomatisations non logicistes des mathématiques, que ce soit celle de Peano, celle de Hilbert ou d'autres.

\section{La vitesse}

L'enfant construit la notion de vitesse antérieurement à celle d'espace et de temps, à partir de l'expérience du dépassement. Ceci rejoint les conceptions de la physique relativiste, à l'encontre de celles de la mécanique newtonnienne, où la vitesse n'est qu'une relation entre l'espace parcouru et le temps et leur est donc notionnellement postérieure. Piaget rappelle que c'est à la suggestion d'Einstein qu'il avait entrepris des recherches sur la genèse de la notion de vitesse, et il souligne qu'en 1954, deux physiciens français, Abelé et Malvaux, ont réussi à refondre les notions fondamentales de la relativité en construisant d'abord la notion de vitesse à partir du dépassement ${ }^{12}$. Il est sans doute intéressant de constater cette concordance entre l'explication génétique et une théorie axiomatisée, mais les remarques faites plus haut concernant

11. Henti Bergson; Essai sur les données immédiates de la conscience, 120e édition, Paris, P.U.F., 1967, pp. $56-78$; L.E.J. BRoUwer, Intuitionism and Formalism in Pbilosopby of Matbematics Selected Readings, by Paul Benacerraf and Hilary Putnam, Englewood Cliffs, Prentice Hall, 1964, p. 69.

12. J. AbelÉ et P. MAlvaux, Vitesse et univers relativiste, Paris, Éditions Sedes, 1954 ; Jean PIAGET, Six études de psychologie, Paris, Gonthier, 1964, pp. 97-100 et 59-61; Psychologie et épistémologie, Paris, Gonthier, 1970, pp. 22-24 et 102-105 ; pour plus de détails,voir aussi les oeuvres suivantes de PIAGET : Le développement de la notion de temps cbez l'enfant, Paris, P.U.F., $1946 ;$ L Les notions de mouvement et de vitesse chez l'enfant, Paris, P.U.F., 1950. 
les rapports entre l'explication génétique et l'axiomatisation resten: valables. La concordance en question ne confère pas à la théorie de la relativité un statut définitif. Elle ne doit pas empêcher qu'une axiomatisation ne cède éventuellement sa place à une autre qui s'avérerait meilleure en termes de simplicité, d'économie conceptuelle, de capacité explicative et prédictive.

\section{E. L'objet permanent}

La permanence de l'objet a été considérée jusqu'au début du siècle comme une notion première et évidente. Ceci a été remis en question par la microphysique : un objet n'existe en tant qu'objet, (plutôt qu'en tant qu'onde), que dans la mesure où il est localisable. La psychologie génétique nous apprend que l'enfant de moins d'une année vit dans un univers de tableaux purement phénoménaux et dénués de permanence puisque son comportement ne présente aucune recherche des objets disparus. Vers l'âge d'un an, l'enfant commence à effectuer cette recherche, mais dans les débuts, il le fait toujours à l'endroit où l'objet a disparu pour la première fois (dans le cas où l'objet est réapparu pour disparaître une seconde fois en un autre endroit) : il est clair que la permanence de l'objet est ici liée à sa localisation.

La permanence de l'objet est chez l'enfant la première forme de l'identité qualitative, elle-même saisie antérieurement à l'identité quantitative, ou conservation des quantités. La psychologie génétique montre que la seconde notion ne dérive pas nécessairement de la première puisqu'elle est acquise par l'intériorisation d'actions réversibles de transvasement de liquides ou de déplacements d'objets. La conservation ne dérive donc pas directement de l'identité comme le croyait Meyerson, mais elle suppose une composition opératoire des transformations qui insèrent l'identité dans le cadre plus large de la réversibilité ${ }^{13}$. Notons-le encore une fois, un tel résultat ne peut être utilisé comme motif valable pour imposer à la science certaines conclusions ou certains procédés, cela pour les raisons énoncées plus haut.

13. Jean PIAGet. Psycbologie et épistémologie, pp. 24-28. Voir aussi les oeuvres suivantes de PIAGET: La construction du réel cbez l'enfant, Neuchâtel, Delachaux et Niestlé, chap. I ; Le développement des quantités chez l'enfant, Delachaux et Niestlé (avec Bärbel INHELDER). 
F. L'espace

Notons enfin que la genèse de l'espace chez l'enfant, aussi bien celle de l'espace perceptif et sensori-moteur, constitué dès les premiers mois de l'existence, que celle de l'espace notionnel et opératoire, commence par un espace topologique où prédominent les relations de voisinage, de continuité, de fermeture et de situation par rapport à une frontière. Viennent ensuite les espaces euclidien et projectif, et finalement l'espace métrique. Ici l'ontogenèse est tout à l'inverse de la genèse historique de la géométrie, qui commence par l'espace métrique ${ }^{14}$. Ceci montre bien que l'idée d'un parallélisme universel et absolu entre l'ontogenèse des notions chez l'enfant et la genèse historique des notions scientifiques n'a aucun fondement, même si un tel parallélisme semble exister (à condition de ne pas trop entrer dans les détails) dans le cas de la causalité analysé plus haut.

\section{QUELQUES AFFIRMATIONS DE PIAGET SUR LES RAPPORTS ENTRE PSYCHOLOGIE GÉNÉTIQUE ET ÉPISTÉMOLOGIE SCIENTIFIQUE}

Que faut-il alors penser de certaines affirmations percutantes de Piaget sur la portée de la psychologie génétique? Citons d'abord deux de ces affirmations:

Cette étude (c'est-à-dire l'étude de la pensée du jeune enfant) montre ensuite comment se construisent les structures cognitives. $\AA$ cet égard la psychologie de l'enfant peut servir de méthode explicative générale en psychologie, car la formation progressive d'une structure constitue à certains égards son explication ${ }^{15}$.

L'étude du mode de construction de certaines structures permet enfin de donner une réponse à certaines questions que se pose la philosophie des sciences : à cet égard la psychologie de l'enfant peut se prolonger en "épistémologie génétique ".16

Dans les pages qui suivent, Piaget tente de justifier la première affirmation en disant que la genèse d'une notion, en tant que portant sur la succession des stades, " rejoint la causalité même des

14. Jean Piaget, Psychologie et épistémologie, pp. 21-22. Pour plus de détails, voir les oeuvres suivantes de PIAGET : La représentation de l'espace chez l'enfant, Paris, P.U.F. (avec Bärbel INHELDER); La géométrie spontanée chez l'enfant, Paris, P.U.F. (avec Bärbel INHELDER et Alina SZEMINSKA).

15. Jean PIAGET, Six études de psychologie, p. 89.

16. Ibidem. 
mécanismes formateurs ${ }^{17}$. Il illustre ensuite sa thèse en invoquant la théorie de la formation du nombre que nous avons exposée plus haut. Mais le problème est qu'une explication génétique, toute valable qu'elle soit, ne peut prétendre être la seule explication possible, ou la seule explication acceptable. Nous ne voyons rien à retrancher de ce que nous avons dit dans les pages précédentes concernant les rapports entre l'explication génétique et l'axiomatisation.

La seconde affirmation est illustrée par l'exemple exposé plus haut des rapports entre, d'une part, la genèse des notions de vitesse et de temps chez l'enfant, et, d'autre part, la physique relativiste. Et Piaget renforce sa thèse initiale en affirmant que la psychologie de l'enfant se prolonge "de façon naturelle ${ }^{18}$ en épistémologie génétique. Il ajoute encore que la pensée du jeune enfant comporte des "résultats positifs qui nous renseignent sur le mode de construction des structures rationnelles et permettent même parfois d'éclairer certains aspects obscurs de la pensée scientifique ${ }^{19}$. Il faut admettre que la connaissance de la genèse constructive des structures logiques et de notions fondamentales comme espace, temps, vitesse, etc., nous aide à mieux voir la relativité de diverses axiomatisations possibles. Mais cette connaissance ne constitue pas un critère absolu qui permette de décider laquelle de ces axiomatisations il faut adopter de préférence. Encore une fois, l'axiomatisation obéit à des impératifs d'économie conceptuelle, de clarté et de simplicité qui ne lui prescrivent pas nécessairement de s'aligner à tout prix sur la genèse des notions, bien que cet alignement ne constitue nullement un mal en soi.

\section{LA PSYCHOLOGIE GÉNÉTIQUE ET LES THÉORIES PHILOSOPHIQUES DE LA CONNAISSANCE}

Les prétentions de Piaget à clarifier certains débats philosophiques concernant la connaissance semblent mieux fondées que ses prétentions à trancher certains débats internes aux mathématiques et aux sciences expérimentales. L'abstraction réfléchissante, la reconstruction périodique de la structure du savoir acquis, le caractère progressif, intégratif et universel des stades de

17. Ibidem, p. 94

18. Ibidem, p. 97

19. Ibidem, p. 100. 
la connaissance enfantine et, enfin, le rôle de l'action dans l'acquisition de la connaissance sont irréconciliables avec certains aspects des théories traditionnelles de la connaissance. Il n'est plus possible, après avoir pris connaissance des études piagétiennes en psychologie génétique, de concevoir l'a priori à la manière de Kant, c'est-à-dire universel, nécessaire et absolu: Piaget nous invite à le relativiser, à le concevoir comme construit et provisoire, comme cadre adéquat où l'on insère les données de l'expérience. On ne peut davantage adhérer aux conceptions empiristes et positivistes classiques, ni accepter une certaine conception populaire de la connaissance comme une pure accumulation de nouvelles données qui s'ajoutent au déjà connu. Pour Piaget, la connaissance implique une action du sujet épistémique sur l'objet et les réactions de ce dernier aux dites actions, une rencontre entre les deux qui a pour effet que le contenu de connaissance qui en résulte porte la marque, en une totalité indissociable, de ses deux sources indispensables.

Piaget n'est certes pas le premier à suggérer de telles critiques. Bachelard et Thomas Kuhn, entre autres, le font de façon percutante. Mais Piaget apporte des arguments nouveaux, issus de la psychologie génétique. Malgré la différence des processus de connaissance analysés respectivement par Kuhn et Piaget, des rapprochements intéressants peuvent être faits sur ce que révèlent leurs analyses concernant le rôle de l'action dans le processus de connaissance, le caractère intégratif des diverses étapes successives de l'évolution de la connaissance et l'incompatibilité de leurs conclusions avec les conceptions idéalistes, empiristes ou positivistes classiques.

\section{L'ÉPISTÉMOLOGIE GÉNÉTIQUE EST-ELLE UNE SCIENCE ?}

Praget a-t-il réussi à construire une épistémologie scientifique, distincte de la philosophie et affranchie de cette dernière? Cette question peut être posée d'abord à propos de la psychologie génétique elle-même. On doit répondre oui dans la mesure où Piaget a développé une bonne méthode pour l'investigation détaillée de l'évolution intellectuelle de l'enfant, et a construit une théorie générale de l'acquisition de la connaissance qui rend compte des découvertes permises par sa méthode. Certains éléments de cette théorie générale, comme par exemple la reconstruction périodique de la structure totale du savoir acquis, la dé- 
pendance du savoir par rapport à un complexe d'actions et de transformations résultant de ces actions, et la succession uniforme des stades, sont clairement falsifiables : leurs conséquences, tout au moins, peuvent être confrontées avec des données expérimentales précises.

Mais il n'est pas certain que tous les items de la théorie piagétienne pourraient être contredits par des contre-exemples spatiotemporellement localisés. C'est le cas, notamment, pour sa conception de l'activité intellectuelle comme un cas particulier de la fonction générale d'adaptation; pour sa définition de l'intelligence comme une tendance ou un effort pour créer un état d'équilibre entre les actions du sujet et celles des objets extérieurs ${ }^{20}$. N'assistons-nous pas ici à un retour subreptice à une certaine notion de "faculté "? Piaget a maintes fois critiqué cette notion et dit qu'il fallait concevoir l'intelligence comme une fonction. Il n'en reste pas moins que ce vocabulaire de "tendance à " et "d'effort pour " nous suggère une notion de faculté (puissance de, capacité á) plus proche de celle d'Aristote que de celle de Kant. Car la faculté intellectuelle a, selon Kant, une structure a priori (les catégories) bien déterminée, ce qui n'est pas le cas pour Aristote.

Notre conviction en ce sens est renforcée par le fait que Piaget extrapole ces notions d'équilibre et d'équilibration hors de la psychologie de l'enfant pour en faire les éléments essentiels d'une définition générale de l'activité intellectuelle. Mais autre est la situation de l'enfant dont l'activité cognitive est mobilisée surtout par un besoin d'adaptation au monde extérieur et autre est la situation du scientifique poursuivant des recherches qui n'ont pas, du moins pour le chercheur lui-même, de but utilitaire immédiat. L'enfant n'a pas le choix quant aux aspects du monde extérieur qui doivent attirer son attention : espace, temps, permanence des objets, causalité, vitesse, etc. Cela lui est imposé à la fois par sa structure sensori-motrice (qui a un caractère standard chez la très grande majorité des sujets) et par ses besoins d'adaptation.

Le scientifique à beaucoup plus de liberté quant au choix des questions sur lesquelles il portera sa curiosité intellectuelle. Les structures logiques sont chez lui plus développées que chez l'enfant ; il a du monde une expérience beaucoup plus diversifiée, et il

20. Jean PIAGET, Six études de psychologie, pp. 114-131 et 172-174. 
peut s'appuyer sur une gamme fort variée de connaissances déjà acquises par ses prédécesseurs. Il peut donc effectuer un choix de questions auxquelles il s'intéresse gratuitement, c'est-à-dire sans y être obligé par des impératifs de conservation, de survie ou de mieux-être qui lui viendraient de sa situation physico-biologique. Évidemment, ce choix peut être limité pratiquement par des circonstances ou des décisions d'ordre idéologique ou politique, par exemple le contrôle des orientations de la recherche par les organismes distributeurs de crédits. Mais il n'en reste pas moins que le scientifique est susceptible de chercher la réponse à une question sans que cela soit lié, pour lui ou pour la société dont il est membre, à la solution de problèmes pratiques perçus comme tels au moment où la recherche est en cours.

On peut donc se demander : quel équilibre Einstein visait-il quand il a cherché une solution à la contradiction qu'il apercevait entre le principe galiléen de relativité et la constance de la vitesse de la lumière? Sans doute peut-on répondre qu'il cherchait un équilibre intellectuel intérieur, ne pouvant plus continuer de vivre, comme physicien, avec cette contradiction ; qu'en remplaçant la conception newtonienne de l'espace par une autre, il s'est accomodé à de nouvelles données expérimentales, soit la constance de la vitesse de la lumière et les résultats des expériences de Michelson et Morley ; et que par sa nouvelle conception de l'espace, il s'est mieux assimilé le monde extérieur, ce qui a créé un nouvel équilibre entre l'ensemble des connaissances théoriques et les faits expérimentaux, d'abord dans la pensée d'Einstein et dans celle de plusieurs autres physiciens. Cet équilibre a été par la suite suivi d'un autre, soit l'équilibre entre les nouvelles conceptions théoriques et certains résultats pratiques, certaines transformations obtenues grâce à leur application: il s'agit d'une nouvelle forme d'équilibre entre l'homme et son milieu.

En ce deuxième sens, l'usage de la notion d'équilibre est acceptable. Mais de l'enfant à Einstein, il se produit un glissement du sens des mots équilibration, équilibre, accomodation et adaptation. Appliqués aux travaux d'Einstein, ils ne vous disent pas davantage que certaines formules du vieil Aristote, formules répétées et commentées par ses commentateurs médiévaux : une question surgit toujours d'un état d'étonnement et de malaise intellectuel face à l'existence de plusieurs hypothèses explicatives irréconciliables à 
propos d'un même phénomène. Suit une recherche qui aboutit parfois à une solution qui élimine toutes les hypothèses sauf une, et qui provoque chez le chercheur un état au moins temporaire de certitude, c'est-à-dire de satisfaction et de plénitude, ce qui met fin à la recherche. Tout effort de définition générale de l'intelligence ou de l'activité intellectuelle sans aucune référence au contexte particulier dans lequel elle s'exerce ne peut aboutir qu'à de vagues généralités. L'activité intellectuelle est trop variée pour qu'on puisse la couler dans une définition qui soit à la fois générale, unique et précise.

Les idées de Piaget sur l'intelligence sont donc philosophiques. Elles ne peuvent faire l'objet d'une falsification au sens précis du terme, c'est-à-dire être contredites par un contre-exemple précisément localisé sur le plan spatio-temporel. Elles constituent une hypothèse ou idée directrice, un cadre général de recherche, un programme d'investigation. Même si elles ne sont pas susceptibles d'une falsification expérimentale au sens strict du mot, il reste possible d'apprécier à long terme la fécondité de ses cristallisations ou incarnations dans des méthodes pédagogiques, dans la pratique scientifique, ou dans tout autre aspect de la culture. Ces appréciations pourront amener des transformations de ces idées philosophiques, ou leur abandon. Les idées philosophiques ou métaphysiques ne sont jamais réfutées. On les abandonne quand elles cessent d'être fécondes pour interpréter les situations vécues et les faire progresser.

Revenons maintenant à notre question sur le caractère scientifique de l'épistémologie et posons-la de nouveau, cette fois-ci à propos de l'épistémologie des sciences. L'oeuvre de Piaget en ce domaine étant beaucoup plus modeste que celle de chercheurs comme Kuhn, Koyré ou Canguilhem, c'est plutôt à propos des travaux de ces derniers qu'il faut poser la question. Il semble bien que, confiée aux soins de ces spécialistes, l'épistémologie scientifique soit en train de devenir une science, puisqu'elle met en oeuvre des méthodes d'analyse de plus en plus adéquates et raffinées pour étudier et comparer des moments différents de la pensée scientifique. Mais cette épistémologie est-elle exempte de postulats et d'interprétations philosophiques? Les auteurs concernés admettent eux-mêmes que le pôle théorique de la science recèle toujours de tels postulats : Kuhn voit en eux une partie essentielle du cadre 
théorique (disciplinary matrix) de la science ${ }^{21}$. L'épistémologie en tant que science ne devrait pas faire exception. C'est le sort commun des sciences particulières d'être enveloppées dans un réseau de principes philosophiques qui ne sont ni consciemment assumés par le scientifique, ni clairement exprimés, ni précisément définis. C'est le rôle du philosophe de formuler ces principes clairement, d'en examiner la portée et les limites, d'en critiquer les contradictions si nécessaire et d'attirer l'attention sur leur caractère problématique et relatif. Le philosophe peut, à l'occasion, rappeler au scientifique qu'un attachement exagéré à ces principes peut inhiber le renouvellement de la pensée scientifique, témoin ces physiciens du début du siècle qui, trop attachés à une problématique kantienne, ont refusé la physique relativiste.

Le philosophe ne doit pas s'affliger de voir un domaine comme l'épistémologie se détacher de la philosophie pour devenir une science autonome. Il doit plutôt en être content, car une information plus riche sur les processus de la connaissance lui sera dorénavant fournie. Rien n'empêche d'ailleurs qu'on soit en même temps épistémologue et philosophe. La science et la philosophie y gagnent toujours à cohabiter dans le même cerveau.

Département de Philosophie, Université de Sherbrooke.

21. Thomas S. KHUN ; The Structure of Scientific Revolutions, Chicago, University of Chicago Press, 2nd Edition, Enlarged, pp. 182-187. 